\title{
POSSIBILITY OF SUPERCONDUCTIVITY OF TWO-DIMENSIONAL ELECTRONS ON THE SURFACE OF LIQUID HELIUM FILMS
}

\author{
B. Tanatar and T. Hakioğlu \\ Department of Physics, Bilkent University, Bilkent, 06533 Ankara, Turkey \\ (Received 31 March 1993; accepted for publication 16 June 1993 by G. Bastard)
}

\begin{abstract}
We consider the possibility of superconductivity in a system of twodimensional electrons on the surface of liquid helium films. Taking into account of the interaction between electrons and the surface excitations of liquid helium films-ripplons, within the weak coupling BCS theory, we estimate the superconducting transition temperature for various interaction strengths, film thicknesses, and electron densities. The superconducting transition temperature $T_{c}$, under experimentally realizable conditions, is calculated to be a few mK's.
\end{abstract}

THE SYSTEM of electrons on the surface of liquid helium, because of its experimental accessibility, has been studied quite intensely. The electrons above the surface falling into the image-potential bound states relative to the motion perpendicular to the surface, form an ideal two-dimensional (2D) electron gas at low temperatures. Their accessibility provides yet another physically realizable $2 \mathrm{D}$ electron gas, along with Si-MOSFETs and $\mathrm{GaAs} / \mathrm{Al}_{x} \mathrm{Ga}_{1-x}$ As heterostructures. Recent interest in this system, both from a theoretical and experimental standpoint, is mainly due to the possibility of observing Wigner transition, and the self-trapping of electrons in the plane parallel to the helium surface.

In the experiments performed [1-3] on bulk liquid helium, electron densities attained are in the range $n \sim 10^{5}-10^{9} \mathrm{~cm}^{-2}$, thus the Fermi energy $\epsilon_{F}$ is much smaller than $k T$ even at very low temperatures. In this classical regime the Wigner crystallization has been observed [2, 3]. Mechanisms for the melting of 2D electron solid in different regimes have been discussed [4].

In order to reach the quantum regime $\left(n>10^{10}\right.$ $\mathrm{cm}^{-2}$ ) several proposals [5] are made, of which the most interesting one uses a thin helium film on top of a metallic substrate to enhance the stabilizing force. Measurements of the shear modulus [6], specific heat [7] and mobility [8] around melting point have been performed. An important characteristic of a system of electrons on a helium film is the flexibility in changing the interaction strength between the electrons. Because of the finite distance between the electron layer and the substrate (viz., helium film thickness) the bare Coulomb interaction is screened by the substrate. Screening can dramatically affect the interparticle potential. For instance, a metallic substrate and helium-film thickness smaller than the average electron-electron distance, results in a dipole-dipole interaction. Many properties of the $2 \mathrm{D}$ electrons formed on the surface of liquid helium has been investigated both theoretically and experimentally. Peeters and Platzman [5, 9] have studied the melting and obtained the phase diagram of this system. An interesting result appears to be the reevaporation of the 2D Wigner crystal at extremely low densities, and at some critical film thickness the prevention of crystallization at all densities, which was also noted by $\mathrm{Ma}$ and Inkson [5].

The motion in the plane of a single electron is free except for coupling to the thermally excited ripples of the liquid surface. This coupling is analogous to the electron-phonon coupling. The interaction between electrons and ripplons originate from the change in energy of the electron as it rides on the surface waves in the presence of an electric field $\mathcal{E}$. The polaron aspects of $2 \mathrm{D}$ electrons on the films of liquid helium were investigated by Jackson and Platzman [10]. The ground-state energy, magnetization, and susceptibility of this system in a perpendicularly applied magnetic field, within the Feynman path-integral formalism, were calculated by Jackson and Peeters [11]. The surface mobility of electrons due to scattering from ripplons and impurities including the quantum degeneracy effects was considered by Pereira et al. [12]. The frequency dependent response, and temperature dependent properties of this elec- 
tron-ripplon system at low temperatures was studied by Peeters and Jackson [13] where they calculated the effect of self-trapping on the mobility spectrum. Several aspects of the dynamical properties of the Wigner lattice in such systems have also been investigated [14]. The status of the field has been surveyed in various reviews [15-18] in detail.

The purpose of this communication is to consider the electron-ripplon interaction in the $2 \mathrm{D}$ electron system on the surface of liquid helium films, for a possible superconducting transition. Our primary aim is to estimate the superconducting transition temperature $T_{c}$ for experimentally accessible system parameters. As has been recently noted [16], the possibility of a simple BCS-like transition has been anticipated $[5,17]$ but no estimate of $T_{c}$ was given in the previous studies. Since the typical phonon energies $[17,18]$ are about $\sim 10^{-2} \mathrm{~K}$, we expect the electron system on the surface of helium films to exhibit superconductivity with $T_{c}$ comparable to these energies.

The dispersion relation for ripplons of a charged helium film of thickness $d$ is given by [19]

$$
\begin{aligned}
\omega_{k}^{2}= & k\left[\left[\frac{3 c}{\rho d^{4}}+g\right]+\frac{\tau}{\rho} k^{2}-\frac{4 \pi \mathrm{e}^{2} n^{2}}{\rho} k \frac{1+\mathrm{e}^{-2 k d}}{1-\mathrm{e}^{-2 k d}}\right] \\
& \times \tanh (k d),
\end{aligned}
$$

where $\rho=0.145 \mathrm{~g} / \mathrm{cm}^{-3}$ is the helium mass density, $g$ the acceleration due to gravity, $\tau=0.378 \mathrm{erg}$ $\mathrm{cm}^{-2}$ the surface tension of the helium film, $n$ the areal density of electrons on the helium surface, and $c=9.5 \times 10^{-15} \mathrm{erg}$ is the van der Waals coupling constant of the liquid helium to the substrate. As discussed by Peeters [9], for electron densities greater than a critical density $n^{*}$, the helium surface becomes unstable. For thin films $(k d \ll 1)$ on metallic substrates, this limiting density is estimated to be [9] $n^{*} \sim 100 / \mathrm{d}^{3 / 2}$, valid for $\mathrm{d}<10^{3} \AA$.

In order to estimate the superconducting transition temperature $T_{c}$ arising from electron-ripplon coupling, we first evaluate the McMillan function [20] $\alpha^{2} F(\omega)$

$$
\alpha^{2} F(\omega)=\sum_{\boldsymbol{k} k^{\prime}}\left|g_{k k^{\prime}}\right|^{2} \delta\left(\omega-\omega_{k-k^{\prime}}\right) \delta\left(\xi_{\boldsymbol{k}}\right) \delta\left(\xi_{\boldsymbol{k}^{\prime}}\right) / \sum_{\boldsymbol{k}} \delta\left(\xi_{\boldsymbol{k}}\right),
$$

where $g_{k k^{\prime}}$ denotes the electron-ripplon matrix elements, and $\xi_{k}=\epsilon_{k}-\epsilon_{F}$ are the electron energies relative to the Fermi energy $\epsilon_{F}$. The electron-ripplon interaction is given by

$\left|g_{k}\right|^{2}=\frac{\hbar k \tanh (\mathbf{k d})}{2 \rho \omega_{k}}(e \mathcal{E})^{2}$.

In the above equation $\mathcal{E}$ is the total holding electric field perpendicular to the surface, and in general has contributions from the externally applied field $\mathcal{E}^{\text {ext }}$, and the image charges in the substrate. Following Jackson and Platzman [10], we linearize the ripplon dispersion relation

$\omega_{k}=\left(\frac{3 c}{\rho d^{4}}+g\right)^{1 / 2} d^{1 / 2} k=s k$

for $k<k_{c}=\sqrt{\frac{\rho d}{\sigma}\left(\frac{3 c}{\rho d^{4}+g}\right)}$,

where we have introduced the propagation velocity $s$ and the cut-off wave vector $k_{c}$. The above approximation is good at $T=0$ and $d \sim 100 \AA[10,13]$. We also introduce the coupling constant $\alpha_{e-r}$ for the electron-ripplon interaction

$\alpha e-r=\frac{(e \mathcal{E})^{2} m d}{4 \pi \hbar^{2} 2}$.

The superconducting coupling constant is determined from

$\lambda=2 \int \mathrm{d} \omega \frac{\alpha^{2} F(\omega)}{\omega}$,

and the transition temperature $T_{c}$ is estimated by solving the gap equation within the BCS theory,

$\frac{1}{\lambda}=\int \frac{\mathrm{d} \epsilon}{\epsilon} \tanh \left(\frac{\epsilon}{2 k_{B} T_{c}}\right)$

We present and discuss our results in the following. First, the McMillan function $\alpha^{2} F(\omega)$ is plotted as a function of $\omega / \sqrt{2 \hbar s k_{F}}$ for the electronripplon system under study in Fig. 1. The $\alpha^{2} F(\omega)$ function exhibits a single peak and a sharp cut-off reflecting the simple model of the electron-ripplon dispersion. The superconducting coupling constant $\lambda$ calculated using the $\alpha^{2} F(\omega)$ function is $\sim 0.3$, validating the BCS-type mechanism.

In Fig. 2 we show the calculated superconducting transition temperature $T_{c}$ as a function of the electron-ripplon interaction strength parameter $\alpha_{\mathrm{e}-\mathrm{r}}$, at the electron density $n=10^{10} \mathrm{~cm}^{-2}$. The solid, dashed and dotted curves indicate the helium film thickness $d=200 a_{B}, 100 a_{B}$, and $20 a_{B}$, respectively, where $a_{B}=\hbar^{2} / m e^{2}$ is the Bohr radius. Here the coupling constant $a_{e-r}$ may be regarded as a measure of the perpendicular electric field $\mathcal{E}$. For $d=100 a_{B}$, the value of $\mathcal{E}$ becomes $\sim 10^{3} \alpha_{\mathrm{e}-\mathrm{r}}^{1 / 2} \mathrm{~V} / \mathrm{cm}^{-1}$ 


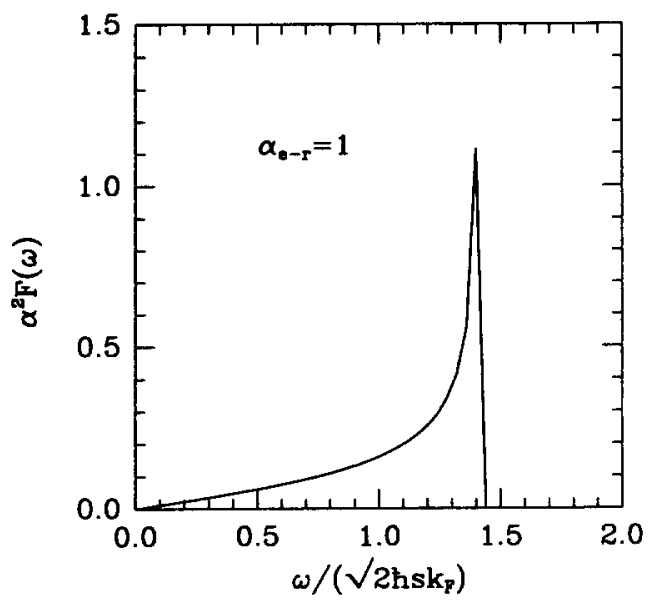

Fig. 1. The McMillan function $\alpha^{2} F(\omega)$ vs $\omega$ for the helium film thickness $d=200 a_{B}$, the electron density $n=10^{10} \mathrm{~cm}^{-2}$, and the electron-ripplon coupling strength $\alpha_{\mathfrak{e}-\mathrm{r}}=1$.

which is of the order reached in the experiments. The electron density dependence of the transition temperature is displayed in Fig. 3. We have chosen the electron-ripplon coupling parameter $\alpha_{\mathrm{e}-\mathrm{r}}=1$. Solid, dashed, and dotted lines indicate the helium film thickness $d=200 a_{B}, 100 a_{B}$, and $20 a_{B}$, respectively. We observe that highest transition temperatures can be attained as $n \sim 10^{12} \mathrm{~cm}^{-2}$ and $d \sim 20 a_{B}$.

We have used the McMillan function $\dot{\alpha}^{2} F(\omega)$ for the electron-ripplon interaction to calculate various moments of the ripplon frequencies as defined by

$\left\langle\omega_{n}\right\rangle=\frac{2}{\lambda} \int \mathrm{d} \omega \omega^{n} \frac{\alpha^{2} F(\omega)}{\omega}$,

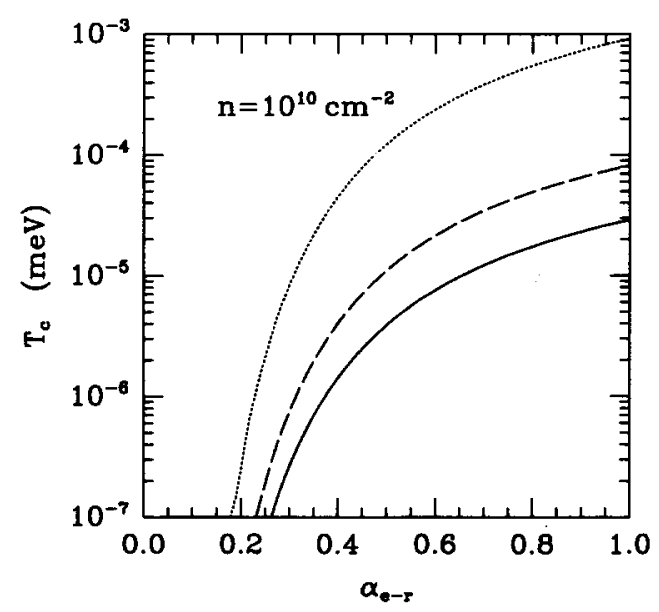

Fig. 2. Superconducting transition temperature $T_{c}$ as a function of the electron-ripplon coupling strength $\alpha_{e-r}$ at the electron density $n=10^{10} \mathrm{~cm}^{-2}$. Solid, dashed, and dotted lines are for the helium film thickness $d=200 a_{B}, 100 a_{B}$, and $20 a_{B}$, respectively. and the logarithmic-mean frequency as given by

$\left\langle\omega_{1 \mathrm{n}}\right\rangle=\exp \left\{\frac{2}{\lambda} \int \mathrm{d} \omega \ln \omega \frac{\alpha^{2} F(\omega)}{\omega}\right\}$.

The first few moments that may be used in the prediction of the transition temperature $T_{c}$ according to various models discussed by Allen and Mitrović [21], are obtained as $\left\langle\omega_{1}\right\rangle=7.25 \times 10^{-4} \mathrm{meV}$, $\left\langle\omega_{2}\right\rangle^{1 / 2}=8.10 \times 10^{-4} \mathrm{meV}$, and $\left\langle\omega_{1 \mathrm{n}}\right\rangle=5.86 \times 10^{-4}$ $\mathrm{meV}$ which are all of the order of cut-off frequency $\sqrt{2} \hbar s k_{F} \sim 8.5 \times 10^{-4} \mathrm{meV}$. Since our main interest was to estimate $T_{c}$ in the spirit of BCS theory, we did not take into account the Coulomb repulsion between the electrons. It may be included in the calculation of $T_{c}$ through the Coulomb pseudopotential $\mu^{*}[21,22]$. Recently, Combescot [22] have studied the exact solution of the Eliashberg equations in the weak coupling limit $\left(\lambda, \mu^{*} \rightarrow 0\right)$ for an arbitrary McMillan function $\alpha^{2} F(\omega)$. We obtain qualitatively similar results using the $T_{c}$ formula proposed by Combescot [22] in which $\left\langle\omega_{\mathrm{ln}}\right\rangle$ plays an important role.

It would be interesting to further study the system of 2D electrons on the surface of liquid helium films through computer simulations. For instance, Path Integral Monte Carlo (PIMC) calculations may be performed to theoretically determine the superconducting transition, similar to the case of superfluidity in liquid helium [23].

In summary, we have investigated the possibility of superconducting state, and in particular the transition temperature $T_{c}$ for $2 \mathrm{D}$ electrons on the surface of liquid helium films interacting with the surface excitations. We find that the electron-ripplon

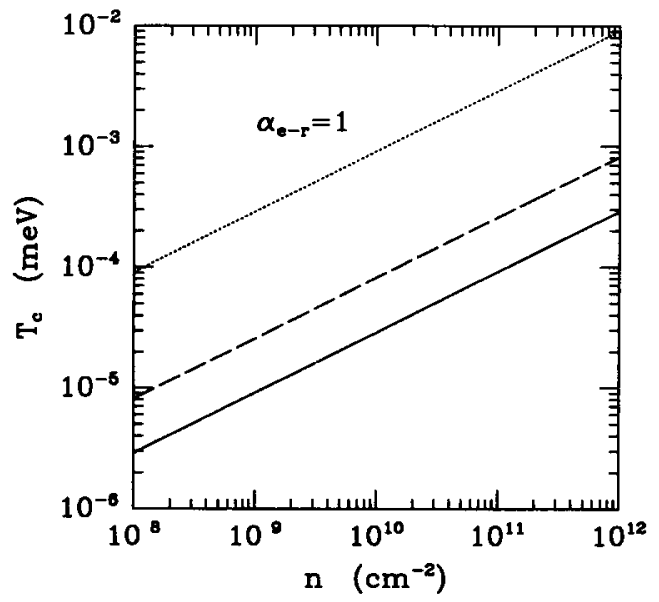

Fig. 3. Superconducting transition temperature $T_{c}$ as a function of the electron density $n$, for the electronripplon coupling strength $\alpha_{\mathrm{e}-\mathrm{r}}=1$. Solid, dashed, and dotted curves are for the helium film thickness $d=200 a_{B}, 100 a_{B}$, and $20 a_{B}$, respectively. 
system undergoes a weakly coupled superconducting transition, and for experimentally accessible parameters $T_{c}$ is of the order of $\mathrm{mK}$, comparable to the typical phonon (ripplon) energies.

Acknowledgement - We would like to thank $\mathrm{Dr}$ M.Z. Gedik for useful discussions and comments.

\section{REFERENCES}

1. C.C. Grimes, Surf. Sci. 73, 379 (1978); F.I.B. Williams, Surf. Sci. 113, 371 (1982).

2. C.C. Grimes \& G. Adams, Phys. Rev. Lett. 42, 795 (1979).

3. E.Y. Andrei, Phys. Rev. Lett. 52, 1449 (1984).

4. P.M. Platzman \& H. Fukuyama, Phys. Rev. B10, 3150 (1974); T. Nagai \& A. Onuki, J. Phys. C11, L681 (1978).

5. F.M. Peeters \& P.M. Platzman, Phys. Rev. Lett. 50, 2021 (1983); K.B. Ma \& J.C. Inkson, $J$. Phys. C11, L411 (1978).

6. G. Deville, A. Valdes, E.Y. Andrei \& F.I.B. Williams, Phys. Rev. Lett. 53, 588 (1984).

7. C.D. Glattli, E.Y. Andrei \& F.I.B. Williams, Phys. Rev. Lett. 60, 420 (1988).

8. M.A. Stan \& A.J. Dahm, Phys. Rev. B40, 8895, (1989); 40, 9006 (1989).

9. F.M. Peeters, Phys. Rev. B30, 159 (1984).

10. S.A. Jackson \& P.M. Platzman, Phys. Rev. B24, 499 (1981); Surf. Sci. 142, 125 (1984).

11. S.A. Jackson \& F.M. Peeters, Phys. Rev. B30, 4196 (1984).
12. M.C. Pereira, G.E. Marques \& N. Studart, Phys. Rev. B46, 1857 (1992).

13. F.M. Peeters \& S.A. Jackson, Phys. Rev. B31, 7098 (1985); 34, 1539 (1986).

14. Z. Lenac \& M. Sunjić, Phys. Rev. B44, 11465 (1991); and references therein.

15. F.M. Peeters, in The Physics of the TwoDimensional Electron Gas (Edited by J. T. Devreese \& F.M. Peeters), Plenum Press, New York (1987).

16. A.J. Dahm, in Excitations in Two-Dimensional and Three-Dimensional Quantum Fluids (Edited by A.F.G. Wyatt \& H.G. Lauter), Plenum Press, New York, (1991).

17. Yu. P. Monarkha \& V.B. Shikin, Fiz. Nizk. Temp. 8, 563 (1982) [Sov. J. Low Temp. Phys. 8, 279 (1982)].

18. V.B. Shikin \& Yu.P. Monarkha, Fiz. Nizk. Temp. I, 957 (1975) [Sov. Phys. Low Temp. Phys. I, 459 (1975)].

19. L.P. Gor'kov \& D.M. Chernikova, Pis'ma $Z h$. Eksp. Teor. Fis. 18, 119 (1973) [JETP Lett. 18, 68 (1973)]; H. Ikezi \& P.M. Platzman, Phys. Rev. B23, 1145 (1981).

20. W.L. McMillan, Phys. Rev. 167, 331 (1986); P.B. Allen \& R.C. Dynes, Phys. Rev. B12, 905 (1975).

21. P.B. Allen \& B. Mitrovic, Solid State Physics 37, 1 (1982).

22. R. Combescot, Phys. Rev. B42, 7810 (1990).

23. D.M. Ceperley \& E.M. Pollock, Phys. Rev. Lett. 56, 351 (1986). 